\title{
Prevalência de bullying em uma amostra representativa de adolescentes brasileiros
}

\author{
Bullying prevalence in a representative sample of Brazilian adolescents \\ Prevalencia de bullying en una muestra representativa de adolescentes brasileños \\ Cléa Adas Saliba GARBIN ${ }^{1}$ \\ Renata Colturato Joaquim GATTO $^{2}$ \\ Artênio José Ísper GARBIN ${ }^{3}$
}
${ }^{1}$ Professora Titular do Programa de Pós-Graduação em Odontologia Preventiva e Social. Faculdade de Odontologia, UNESP Univ. Estadual Paulista, 16015-050 Araçatuba - SP, Brasil
${ }^{2}$ Doutoranda, Programa de Pós-Graduação em Odontologia Preventiva e Social. Faculdade de Odontologia, UNESP Univ. Estadual Paulista, 16015-050 Araçatuba - SP, Brasil
Professor Adjunto do Programa de Pós-graduação em Odontologia Preventiva e Social. Faculdade de Odontologia, UNESP Univ. Estadual Paulista, 16015-050 Araçatuba - SP

\begin{abstract}
Resumo
Introdução: Bullying é considerado um subtipo de violência e caracteriza-se por ações negativas de parte de um ou mais estudantes em uma relação desigual de poder. Objetivo: verificar a prevalência do Bullying em uma amostra representativa de adolescentes brasileiros. Metodologia: Estudo epidemiológico transversal de base escolar. Participaram da pesquisa, adolescentes de 11 a 16 anos, matriculados na rede pública de ensino, de um município de médio porte do noroeste paulista. A prática de bullying foi avaliada utilizando o questionário Kidscape. A direção da escola intermediou a autorização dos pais/responsáveis para que estes assinassem o termo de consentimento livre e esclarecido, permitindo assim a participação de seus filhos na pesquisa. Os dados foram analisados por meio de análise estatística descritiva e os resultados foram expressos em valores absolutos e relativos. Resultados: no total, 815 adolescentes foram avaliados. A maioria pertencia ao sexo feminino $(59,9 \%)$ e tinha a cor da pele parda $(47,2 \%)$. Quase metade $(48,22 \%)$ dos adolescentes participantes da pesquisa relatou já ter sido vítima de bullying. A forma mais prevalente de intimidação sofrida foi a verbal $(46,56 \%)$ e os episódios ocorreram principalmente dentro da sala de aula $(45,55 \%)$. A maioria dos agressores eram meninos $(65,14 \%)$. Grande parte dos estudantes $(65,2 \%)$ relatou ter sido vítima de bullying mais de uma vez e nunca atuaram como intimidadores $(67,7 \%)$. Entre os participantes, $46,56 \%$ acreditam que a culpa pelos episódios de bullying seja do próprio agressor. Conclusão: houve uma alta taxa de prevalência de adolescentes vítimas de bullying na população estudada.
\end{abstract}

Descritores: Bullying; Adolescente; Violência.

\begin{abstract}
Introduction: bullying is considered a subtype of violence and is characterized by negative actions from one or more students in an unequal power relationship. Objective: To determine the prevalence of bullying in a representative sample of Brazilian adolescents. Methodology: cross-sectional epidemiological study of school-based. Participated in the survey, adolescents 11-16 years, enrolled in the public school system, a medium-sized municipality in the Northwest of São Paulo. The bullying practice was assessed using the questionnaire Kidscape. The school administration mediated permission from parents / guardians so that they sign Informed Consent, allowing the participation of children in research. Data were analyzed by descriptive statistical analysis and the results were expressed in absolute and relative values. Results: A total of 815 adolescents were evaluated. Most were females (59.9\%) and brown skinned $(47.2 \%)$. Almost half $(48.22 \%)$ of the adolescents reported having been a victim of bullying. The most prevalent form of suffering intimidation was verbal (46.56\%) and the episodes occurred primarily within the classroom (45.55\%). Most of the attackers were boys $(65.14 \%)$. Most students $(65.2 \%)$ reported being bullied more than once and never acted like bullies (67.7\%). Among the participants, $46.56 \%$ believe that the blame for bullying episodes is the assailant. Conclusion: There was a high prevalence of adolescent victims of bullying in the studied population.
\end{abstract}

Descriptors: Bullying; Adolescent; Violence.

\section{Resumen}

Introducción: el bullying es considerado un subtipo de violencia y se caracteriza por acciones negativas de parte de uno o más estudiantes en una relación desigual de poder. Objetivo: verificar la prevalencia de bullying en una muestra representativa de adolescentes brasileños. Metodología: estudio epidemiológico transversal con escolares. Participaron de la investigación, adolescentes de 11 a 16 años matriculados en la red pública de enseñanza, de una ciudad mediana del noroeste paulista. La práctica del bullying fue evaluada usando el cuestionario Kidscape. Las directivas de la escuela intermediaron entre los investigadores y padres/responsables para la firma de la Declaración de Consentimiento Informado, con el fin de permitir la participación de sus hijos en la investigación. Se realizó un análisis estadístico descriptivo de los datos y presentados en valores absolutos y relativos. Resultados: en total, 815 adolescentes fueron evaluados. La mayoría era de mujeres (59,9\%) con color de piel parda $(47,2 \%)$. Casi la mitad $(48,22 \%)$ de los adolescentes que participaron de la investigación relataron haber sido víctima de bullying. La forma más frecuente fue el acoso verbal $(46,56 \%)$ e os episodios ocurrieron principalmente dentro de las salas de aula. La mayoría de los agresores eran hombres $(65,14 \%)$. Gran parte de los estudiantes $(65,2 \%)$ relató haber sido víctima de bullying más de una vez y que nunca habían actuado como intimidadores $(67,7 \%)$. Entre los participantes, el 46,56\% creen que la culpa de los episodios de bullying es del propio agresor. Conclusión: se identificó una alta tasa de prevalencia de adolescentes víctimas de bullying en la población estudiada.

Descriptores: Acoso Escolar; Adolescente; Violencia. 


\section{INTRODUÇÃO}

A adolescência é caracterizada por grandes transformações e o início dessa fase é marcado pelas transformações corporais (puberdade) ${ }^{1}$. A construção da identidade nesse período pode receber a influência de fatores intrapessoais (aptidões próprias e características adquiridas de personalidade), de fatores interpessoais (identificações com outrosindivíduos) e de fatores culturais (valores sociais do meio em que se vive). ${ }^{1}$ Dessa forma, as relações de amizade tem forte influencia na vida dos adolescentes, a opinião de amigos sobre a maneira de se vestir, os relacionamentos amorosos e eventos sociais por vezes é superestimada ${ }^{2}$.

No entanto, quando o relacionamento com colegas é permeado por humilhações e violência, uma séria de transtornos emocionais e comportamentais podem ser desencadeados ${ }^{3}$. O bullying, é considerado um subtipo de violência e vem sendo amplamente discutido no meio científico.Trata-se de um comportamento agressivo e persistente, aparentemente sem motivo, com intenção de causar dano físico ou moral em um ou mais estudantes que são mais fracos e incapazes de se defenderem. Nesse cenário, existe uma relação de desigualdade de poder entre os pares ${ }^{4}$.

O bullying pode ser caracterizado por três tipos de agressões: físicas diretas; verbais diretas; e indiretas ${ }^{5-7}$. A agressão física direta envolve ações individuais ou em grupo contra uma única pessoa, através de agressões com socos, tapas, empurrões, chutes, apropriação ou danificação de pertences da vítima. A agressão verbal direta engloba insultos em público (xingamentos, provocações, ameaças, ridicularização, apelidos perversos). E a agressão indireta se dá pelo isolamento e exclusão social dentro do grupo de convivência ${ }^{7}$.

Desse modo, pode-se dizer que a prática de violência verbal ou física no ambiente escolar, sempre existiu, mas atualmente suas consequências vêm sendo discutidas no âmbito da saúde pública ${ }^{8}$, pois as consequências desses episódios podem interferir no desenvolvimento psicossocial e comportamental dos indivíduos envolvidos.

As vítimas de bullying comumente apresentam características como: insegurança, ansiedade, depressão, solidão, infelicidade, sintomas físicos e mentais, e baixa autoestima ${ }^{9}$. A frequência dos episódios repercute muitas vezes em comportamento social inibido, passivo ou submisso. Os envolvidos são mais vulneráveis, sentem medo, vergonha e a autoestima cada vez mais baixa podendo culminar na vitimização continuada.

Além das interferências emocionais provocadas nas vítimas, o bullying também pode ser visto como fator de risco para os agressores no que diz respeito à delinquência juvenil e à criminalidade adulta ${ }^{10}$, sendo imprescindível diagnosticar e elaborar mecanismos de enfrentamento para essas problemáticas nos seus estágios iniciais de desenvolvimento, evitando assim seu agravamento no futuro. Neste contexto, foi objetivo deste trabalho, verificar a prevalência de bullying em uma amostra representativa de adolescentes brasileiros, matriculados na rede pública de ensino.

\section{MATERIAL E MÉTODO}

\section{$>$ DESENHO DO ESTUDO}

Trata-se de um estudo epidemiológico transversal realizado com adolescentes, matriculados na rede pública de ensino do município de Araçatuba, SP, no ano de 2014.

O município possui 20 escolas de ensino fundamental na área urbana. Participaram da pesquisa 19 escolas, pois uma escola não consentiu em participar da pesquisa em tempo hábil para a coleta dos dados $(\mathrm{N}=4.283)$. Os escolares participantes da pesquisa pertenciam à faixa etária de 11 a 16 anos $\left(7^{\circ}, 8^{\circ}\right.$ e $9^{\circ}$ ano do ensino fundamental). A escolha dessa faixa etária teve como justificativa o mínimo necessário de escolarização para responder ao questionário autoaplicável. Não participaram da pesquisa: adolescentes cujos pais não autorizaram a participação; adolescentes que tinham autorização, mas não quiseram participar da pesquisa; e adolescentes que não estavam presentes nas três datas programadas para a coleta dos dados. A amostra final foi composta por 815 adolescentes.

\section{$>$ COLETA DE DADOS}

Para avaliação de bullying entre os escolares, utilizou-se um questionário, Modelo Kidscape, desenvolvido pela instituição inglesa homônima, que atua na prevenção do bullying e do abuso sexual infantil. ${ }^{11} \mathrm{O}$ instrumento é composto por 12 questões de múltipla escolha, sendo que a primeira questão avalia se o adolescente já foi vítima de bullying, aqueles que responderam negativamente, foram desencorajados a continuar preenchendo as demais variáveis, tendo em vista que todas elas abordam os episódios de bullying.

A aplicação do questionário foi realizada na própria instituição de ensino por um único pesquisador, previamente calibrado. $\mathrm{O}$ estudo piloto antecedeu a pesquisa para testar a adequação do instrumento de coleta.

\section{$>$ ANÁLISE DOS DADOS}

Os dados foram analisados utilizando o programa estatístico Epi-info versão 3.5.2. Foi realizada a análise descritiva dos dados, e os resultados foram expressos em tabelas, contendo os valores absolutos e relativos.

\section{$>$ ASPECTOS ÉTICOS}

Este estudo foi aprovado pelo Comitê de Ética em Pesquisa da Faculdade de odontologia de Araçatuba da Universidade Estadual Paulista "Júlio de Mesquita Filho", sob o número de protocolo FOA-01080/2011. Os adolescentes foram abordados apenas quando o termo de consentimento havia sido assinado pelos pais ou 
responsáveis. A coleta teve início após a autorização da dirigente regional de ensino e da direção das escolas envolvidas.

\section{RESULTADOS}

No total, foram distribuídas 4.283 autorizações, para os pais/responsáveis pelos adolescentes, por intermédio da diretoria das escolas. A taxa de retorno foi de $19,03 \%$, totalizando 815 adolescentes participantes.

Os estudantes em sua maioria eram do sexo feminino $488(59,88 \%)$ e $327(40,12 \%)$ eram do sexo masculino. Houve um equilíbrio entre a distribuição da faixa etária: $254(31,17 \%)$ tinham 12 anos, 225 $(27,61 \%)$, tinham 13 anos, 259 (31,78\%) tinham 14 anos, já as idades 11,15 e 16 anos, foram as menos prevalentes com $1,72 \%, 6,75 \%$ e $0,98 \%$ respectivamente. A cor da pele foi autorrelatada pelos estudantes, houve uma predominância da cor da pele parda $385(47,24 \%)$, seguida por branca $333(40,86 \%)$ e negra 97 (11,9\%).

A Tabela 1 apresenta o perfil dos estudantes que sofreram ou não bullying, distribuídos por idade, sexo e cor da pele.

Tabela 1. Descrição do perfil dos adolescentes participantes da pesquisa (idade, sexo e cor da pele), vítimas e não vítimas de bullying. Araçatuba-SP, 2014

\begin{tabular}{|c|c|c|c|c|}
\hline & \multicolumn{2}{|c|}{ Vítima de bullying } & \multicolumn{2}{|c|}{ Não vítima de bullying } \\
\hline VARIÁVEIS & $\mathbf{N}$ & $\%$ & $\mathbf{N}$ & $\%$ \\
\hline \multicolumn{5}{|l|}{ Idade } \\
\hline 11 & 08 & 2,03 & 06 & 1,42 \\
\hline 12 & 128 & 32,58 & 127 & 30,10 \\
\hline 13 & 108 & 27,48 & 117 & 27,72 \\
\hline 14 & 120 & 30,53 & 138 & 32,70 \\
\hline 15 & 26 & 6,62 & 29 & 6,88 \\
\hline 16 & 03 & 0,76 & 05 & 1,18 \\
\hline Total & 393 & 100 & 422 & 100 \\
\hline \multicolumn{5}{|l|}{ Sexo } \\
\hline Feminino & 245 & 62,34 & 244 & 57,82 \\
\hline Masculino & 148 & 37,66 & 178 & 42,18 \\
\hline Total & 393 & 100 & 422 & 100 \\
\hline \multicolumn{5}{|l|}{ Cor da pele } \\
\hline Branco & 161 & 40,97 & 172 & 40,76 \\
\hline Negro & 39 & 9,92 & 58 & 13,74 \\
\hline Pardo & 193 & 49,11 & 192 & 45,50 \\
\hline Total & 393 & 100 & 422 & 100 \\
\hline
\end{tabular}

A Tabela 2 apresenta os dados do questionário Kidscape, que foi adaptado para o presente estudo. Para a questão referente ao sexo do autor do bullying, foram acrescentadas as seguintes categorias: menino, menina e não respondeu; para a questão sobre o tipo de bullying sofrido, foram incluídas: mais de uma resposta e não respondeu. Os fatores associados aos episódios de bullying, estão distribuídos na Tabela 2. Nota-se uma alta prevalência de adolescentes que relataram já terem sido vítimas de bullying, na população estudada. Além disso, também é possível observar que a maioria dos adolescentes sofreu mais de uma vez esse tipo de violência.

Os dados relacionados aos sentimentos das vítimas sobre os episódios de bullying e se alguma vez elas já foram autoras de agressões estão expostos na Tabela 3. A maioria dos adolescentes relatou que se sentiu mal, teve medo ou não quis mais ir para a escola após sofrer bullying.

Tabela 2. Distribuição absoluta e percentual das variáveis relacionadas aos episódios de bullying em adolescentes matriculados na rede pública de ensino. Araçatuba-SP, 2014

\begin{tabular}{|c|c|c|}
\hline VARIÁVEIS & $\mathbf{n}$ & $\%$ \\
\hline \multicolumn{3}{|l|}{ Vítima de bullying } \\
\hline Sim & 393 & 48.22 \\
\hline Não & 422 & 51.78 \\
\hline \multicolumn{3}{|l|}{ Tipo de bullying sofrido } \\
\hline Emocional & 36 & 9.17 \\
\hline Verbal & 183 & 46.56 \\
\hline Físico & 55 & 14.00 \\
\hline Racista & 48 & 12.21 \\
\hline Sexual & 11 & 2.80 \\
\hline Mais de uma resposta & 58 & 14.80 \\
\hline Não respondeu & 2 & 0.21 \\
\hline \multicolumn{3}{|c|}{ Último episódio de bullyingsofrido } \\
\hline Hoje & 35 & 8.91 \\
\hline Nos últimos 30 dias & 84 & 21.37 \\
\hline Nos últimos 6 meses & 71 & 18.07 \\
\hline Há 1 ano ou mais & 203 & 51.65 \\
\hline \multicolumn{3}{|c|}{$\begin{array}{l}\text { Local de ocorrência dos episódios de } \\
\text { bullying }\end{array}$} \\
\hline Indo ou vindo da escola & 21 & 5.34 \\
\hline No refeitório & 7 & 1.78 \\
\hline No banheiro & 6 & 1.53 \\
\hline No pátio & 76 & 19.34 \\
\hline Na sala de aula & 179 & 45.55 \\
\hline Em mais de 1 local & 25 & 6.36 \\
\hline Em outro lugar & 79 & 20.10 \\
\hline \multicolumn{3}{|c|}{ Frequência dos episódios de bullying } \\
\hline Uma vez & 137 & 34.86 \\
\hline Diversas vezes & 161 & 40.97 \\
\hline Quase todos os dias & 57 & 14.50 \\
\hline Várias vezes por dia & 38 & 9.67 \\
\hline \multicolumn{3}{|l|}{ Consequências do bullying } \\
\hline Não teve consequências & 214 & 54.44 \\
\hline Algumas consequências ruins & 140 & 35.61 \\
\hline Consequências terríveis & 22 & 5.96 \\
\hline Fez mudar de escola & 17 & 4.31 \\
\hline \multicolumn{3}{|l|}{ Sexo do agressor } \\
\hline Menino & 256 & 65.14 \\
\hline Menina & 91 & 23.16 \\
\hline Menino e menina & 44 & 11.20 \\
\hline Não respondeu & 2 & 0.51 \\
\hline
\end{tabular}

Tabela 3. Distribuição absoluta e percentual das variáveis relacionadas aos sentimentos das vítimas em relação aos episódios de bullying. Araçatuba-SP, 2014

\begin{tabular}{l|rr}
\multicolumn{1}{c}{ VARIÁVEIS } & n & $\%$ \\
\hline Vítima já foi autor de bullying & 127 & 32.31 \\
Sim & 266 & 67.69 \\
Não & 76 & 19.34 \\
Sentimento da vítima após sofrer bullying & 204 & 51.91 \\
Não me incomodou & 49 & 12.46 \\
Me senti mal & 62 & 16.03 \\
Fiquei com medo / me senti assustado & 1 & 0.25 \\
Não queria mais ir pra escola & & \\
Não respondeu & 13 & 3.31 \\
Sentimento da vítima em relação ao agressor & 147 & 37.40 \\
Gosto deles & 170 & 43.26 \\
Não gosto deles & 62 & 15.78 \\
Tenho pena deles & 1 & 0.25 \\
Não penso nada & & \\
Não respondeu & & \\
De quem é a culpa dos episódios de bullying, & 183 & 46.56 \\
segundo as vítimas & 36 & 9.16 \\
De quem agride & 47 & 11.96 \\
De quem é agredido & & \\
Dos outros alunos que só assistem e não falam & 15 & 3.82 \\
nada & 8 & 2.04 \\
Da direção da escola & 83 & 21.12 \\
Dos professores & 20 & 5.09 \\
Dos pais dos agressores & 1 & 0.25 \\
Mais de 1 resposta & & \\
Não respondeu & &
\end{tabular}

\section{DISCUSSÃO}

Os resultados desta pesquisa são de grande relevância tendo em vista a alta prevalência de bullying entre os adolescentes estudados. Dos 815 alunos 
participantes, $393(48,22 \%)$ responderam que já sofreram agressão/assédio. Existe uma divergência entre estudos nacionais no que diz respeito à taxa de prevalência do bullying. O estudo realizado por Rech et al. ${ }^{8}$ na cidade de Caxias do Sul, RS, abordaram 1.230 escolares dos quais 10,2\% relatam já terem sido vítimas de bullying, o método de avaliação foi o mesmo do presente estudo, o que demonstra a disparidade entre as populações estudadas. A Pesquisa Nacional de Saúde do Escolar (PeNSE), realizada pelo Ministério da Educação em parceria com o IBGE, monitora a saúde dos escolares brasileiros, e inclui, entre outros temas a questão da violência e do bullying nas instituições de ensino nacionais. Em 2012, na sua segunda edição, foram selecionadas escolas públicas e privadas das 26 capitais e do Distrito Federal, entre os escolares participantes 7,2\% relataram sofrer agressão/assédio ${ }^{12}$. Já no estudo de Nascimento et al. ${ }^{13}$, na cidade de Cruz Alta - RS, realizado com 459 estudantes na faixa etária dos 8 aos 14 anos, a taxa de prevalência de bullying foi de $30 \%$, e o instrumento utilizado também foi o Kidscape, demonstrando um resultado mais próximo aos achados deste estudo.

As divergências encontradas entre os estudos podem ser explicadas pelas diferenças regionais e culturais inerentes aos locais de estudo, tendo em vista que o município eleito para a realização desta pesquisa é considerado violento, sendo frequentemente divulgados na mídia local casos de violência nas escolas da cidade.

Houve uma distribuição equilibrada entre os estudantes que sofreram e os que não sofreram bullying no que diz respeito à idade, cor da pele e sexo (Tabela1). No entanto vale ressaltar que a maioria das vítimas foram meninas, o que difere de outros estudos nos quais os meninos são mais assediados ou agredidos ${ }^{12,14}$. Já o sexo do agressor foi predominantemente masculino, o que corrobora com outros achados na literatura ${ }^{8,13}$. Esse achado é importante, pois ressalta a violência de gênero tendo início já na adolescência, onde a mulher encontrase na posição de vítima e o homem como agressor.

A agressão verbal foi a mais prevalente, e pode ser explicado pelo fato das meninas serem as principais vítimas, pois muitos autores relatam que os meninos sofrem mais violência física direta, enquanto as meninas são as principais vítimas de agressões verbais e exclusão ${ }^{8,14,15}$.

A maioria dos estudantes revelou que não tiveram consequências relacionadas aos episódios de agressão. Esse fato pode ter sido amenizado pela questão do tempo, uma vez que a maioria respondeu que os episódios ocorreram há mais de um ano, o que pode gerar um viés de memória. Mais da metade dos envolvidos revelaram que sentiram-se mal após sofrer o assédio, esse mesmo sentimento, também foi predominante no estudo de Nascimento et al. ${ }^{13}$, o que demonstra que o bullying afeta negativamente a vida de quem é assediado.

No que diz respeito à frequência com que os episódios ocorreram, a grande maioria sofreu mais de uma vez com esse tipo de agressão/assédio, fato este que caracteriza o bullying, onde a vítima é perseguida pelo agressor, ocorrendo os episódios repetidas vezes ${ }^{4}$.

Um dado que chama atenção, é o local onde ocorreram as agressões, em quase metade dos casos $(45,55 \%)$ a sala de aula foi o palco desses episódios. Esse fato também foi encontrado nos estudos de Francisco e Libório ${ }^{16}$, Nascimento et al. ${ }^{13}$, Rech et al. ${ }^{8}$ e Vieira et al. ${ }^{15}$, e coloca os professores em uma posição de impotência ou omissão diante do fato. No entanto, quase a totalidade dos alunos não culpa os professores ou a direção da escola por esses acontecimentos, menos de $6 \%$ acreditam que estes sejam culpados. Os alunos acreditam que o principal culpado seja o próprio agressor $(46.56 \%)$ ou os pais dos agressores $(21,12 \%)$. Isso mostra o importante papel da família na visão desses jovens, mas não exime a responsabilidade das instituições de ensino. A escola é um ambiente de grande valor social, já que lida com a produção de conhecimento e com desenvolvimento intelectual e humano dos sujeitos ${ }^{17}$. Poucos estudos revelam o papel dos professores diante do fenômeno da violência nas escolas, Silva e Costa ${ }^{17}$ realizaram um estudo qualitativo com professores da rede pública de ensino e seu papel diante da violência e relataram que os professores fizeram referência ao medo de se tornar uma vítima fatal da violência escolar. Esse fato inibe a motivação que teriam em tentar fazer algo contra ou a favor dos atos de violência.

Quase 70\% dos adolescentes entrevistados, que foram vítimas de bullying, disseram nunca ter agido como autores de agressões/assédio com seus pares. O que difere dos estudos de Moura et al. ${ }^{14}$ e Undheim e Sund ${ }^{18}$, nos quais a maioria das vítimas também se declararam autores de atos agressivos. No entanto, o que difere a violência entre adolescentes e o bullying é justamente o fato das vítimas não reagirem contra seus agressores, ou seja, existe uma relação desigual de poder, na qual a vítima apresenta um comportamento mais reprimido, que difere do comportamento agressivo dos abusadores.

Diante do exposto, é possível observar que o bullying é uma realidade presente em um ambiente que deveria ser um espaço para o desenvolvimento social positivo, para o contato com novas culturas, para agregar valores éticos e para desenvolver o senso crítico, diante do diálogo, no reconhecimento e acedência da diversidade. No entanto, a violência que permeia esse ambiente, compromete a aprendizagem e a qualidade do ensino $^{17}$.

As consequências desses episódios podem 
interferir na vida adulta tanto das vítimas quanto dos agressores. Para as vítimas não são raros os quadros de depressão, angústia, baixa autoestima, estresse, e evasão escolar, podendo até mesmo culminar em atitudes de autoflagelação e suicídio. Já os agressores podem adotar comportamentos de risco, atitudes delinquentes ou tornarem-se ainda mais violentos $8,10,13,19$.

O presente estudo apresenta limitações, como viés de memória, pois os alunos foram questionados sobre episódios de bullying sem limitar o período em que esse fato aconteceu, além disso, trata-se de um estudo transversal, realizado em apenas um município, não podendo estender seus resultados para outras regiões.

Para complementar e aprofundar os achados desta pesquisa sugere-se como estudos futuros a utilização da abordagem qualitativa, para compreender os anseios e sentimentos dos adolescentes vítimas de bullying, bem como a abordagem longitudinal, para que se entenda melhor as consequências desses episódios na vida adulta.

\section{CONCLUSÃO}

Conclui-se que houve uma alta prevalência de bullying entre os adolescentes estudados, sendo a maioria das vítimas meninas e os agressores meninos. As vítimas se declararam não autoras de bullying e a principal agressão sofrida foi a verbal.

\section{REFERÊNCIAS}

1. Schoen-Ferreira TL, Aznar-Farias M, Silvares EF de M. A construção da identidade em adolescentes: um estudo exploratório. Est Psicologia. 2003; 8(1):10715

2. Sebald, H. Adolescents peer orientation: changes in the support system during the past three decades. Adolescence, 1989 Winter; 24(96):937-46.

3. Assis SG, Avanci, JQ, Pesce RP, Deslandes SF. Superação de dificuldades na infância e adolescência: conversando com profissionais de saúde sobre resiliência e promoção da saúde. Rio de Janeiro: FIOCRUZ/ENSP/CLAVES/CNPq; 2006.

4. Olweus D. Bullying at school: basic facts and effects of a school based intervention program. J Child Psychol Psychiatry. 1994 Oct; 35(7):1171-90.

5. Smith PK, Mahdavi J, Carvalho M, Fisher S, Russel S, Tippett N. Cyberbullying: its nature and impact in secondary school pupils. J Child Psychol Psychiatry. 2008 Apr, 49(4):376-85.

6. Craig W, Harel-Fisch Y, Fogel-Grinvald H, Dostaler $\mathrm{S}$, Hetland J, Simons-Morton B, et al. A crossnational profile of bullying and victimization among adolescents in 40 countries. Int J Public Health. 2009 Sep; 54(2):216-24.

7. Zequinão MA, Medeiros P, Pereira B, Cardoso FL. Bullying escolar: um fenômeno multifacetado. Educ Pesqui. 2016; 42(1):181-98.
8. Rech RR, Halpern R, Tedesco A, Santos DF. Prevalence and characteristics of victims and perpetrators of bullying. J Pediatr (Rio J). 2013 MarApr; 89(2):164-70.

9. Nansel TR, Overpeck M, Pilla RS, Ruan WJ, Simons-Morton B, Scheidt P. Bullying behaviors among US youth: prevalence and association with psychosocial adjustment. JAMA. 2001; 285(16):2094-100

10. Silva JL, Oliveira WA, Bono EL, Dib MA, Bazon MR, Silva MAI. Associações entre Bullying Escolar e Conduta Infracional: Revisão Sistemática de Estudos Longitudinais. Psic: Teor e Pesq. 2016 JanMar, 32(1):81-90.

11. Kidscape: preventing bullying, protect in children. Disponível em: http://www.kidscape.org.uk

12. Malta D.C, Prado RR do, Dias AJ, Mello FC, Silva MA, da Costa MR, et al. Bullying and associated factors among Brazilian adolescents: analysis of the National Adolescent School-based Health Survey (PeNSE 2012). Rev Bras Epidemiol. 2014; 17(Suppl 1):131-45

13. Nascimento KB do; Krug M de R; Costa FTL da; Nascimento BB. Bullying entre escolares: um estudo descritivo na cidade de Cruz alta/RS. Reflexão e Ação. 2013; 21(1):196-218.

14. Moura DR de; Cruz, ACN; Quevedo, L de Á. Prevalência e características de escolares vítimas de bullying. J Pediatr (Rio J). 2011 Jan-Feb; 87(1):1923.

15. Vieira IS, Torales APB, Vargas MM, Oliveira CCC. Atitudes de alunos espectadores de práticas de bullying na Escola. Cienc Cuid Saude 2016 Jan/Mar; 15(1):163-70

16. Francisco MV, Libório RM. A study on bullying victimization among peers in elementary and junior high school. Psicol Reflex Crit. 2009;22:200-7

17. Silva MK, Costa BBA. Violência na escola: sentidos e estratégias docentes no contexto da prática. Recei. 2015 Set; 1(2):201-10.

18. Undheim AM, Sund AM. Prevalence of bullying and aggressive behavior and their relationship to mental health problems among 12- to 15-year-old Norwegian adolescents. Eur Child Adolesc Psychiatry. 2010 Nov, 19(11):803-11.

19. Lopes Neto AA. Bullying: comportamento agressivo entre estudantes. J Pediatr (Rio J). 2005 Nov; 81(5 Suppl):S164-72. 


\section{CONFLITO DE INTERESSES}

Os autores declaram não haver conflitos de interesse.

\section{AUTOR PARA CORRESPONDÊNCIA}

Cléa Adas Saliba Garbin

cgarbin@foa.unesp.br

Submetido em 15/08/2016

Aceito em 19/09/2016 